Annales Academiæ Scientiarum Fennicæ

Mathematica

Volumen 38, 2013, 455-471

\title{
ON BANK-LAINE TYPE FUNCTIONS
}

\author{
Jianming Chang* and Yuefei Wang ${ }^{\dagger}$ \\ Changshu Institute of Technology, Department of Mathematics \\ Changshu, Jiangsu 215500, P. R. China; jmchang@cslg.edu.cn \\ Chinese Academy of Sciences, AMSS, Institute of Mathematics \\ Beijing 100190, P. R. China; wangyf@math.ac.cn
}

\begin{abstract}
We continue our previous study on the Bank-Laine type functions: meromorphic functions $f$ that satisfy $f(z)=0 \Longleftrightarrow f^{\prime}(z) \in\{a, b\}$ on the plane, where $a, b$ are two distinct nonzero values. Using quasi-normality, we prove that there is no transcendental meromorphic function with this property when the quotient $a / b$ is a positive integer. Moreover, we prove a quasi-normal criterion for families of such functions. This completes our previous results.
\end{abstract}

\section{Introduction}

A Bank-Laine function $f$ is an entire function that has the following property: $f(z)=0 \Longrightarrow f^{\prime}(z) \in\{-1,1\}$. The Bank-Laine functions arise in connection with solutions of second order homogeneous linear differential equations [1], (see also [6]). In our previous paper [2], we studied the meromorphic functions $f$ on the plane C that satisfy $f(z)=0 \Longleftrightarrow f^{\prime}(z) \in\{a, b\}$, where $a, b$ are two distinct nonzero values. We call such functions Bank-Laine type functions. We constructed there some transcendental meromorphic functions with this property when the quotient $a / b$ is a negative rational number, and proved the following results.

Theorem 1.1. For two distinct nonzero values $a$ and $b$ that satisfy $a / b \in \mathbf{N}$ (positive integers), there is no transcendental meromorphic function $f$ of finite order that satisfies $f(z)=0 \Longleftrightarrow f^{\prime}(z) \in\{a, b\}$.

A rational function $f$ satisfying $f(z)=0 \Longleftrightarrow f^{\prime}(z) \in\{a, b\}$ exists if and only if $a / b$ or $b / a$ is an integer. In fact, all such rational functions have been classified completely [2, Lemma 10, Lemma 11].

It remains a question: whether there are transcendental meromorphic functions $f$ of infinite order that satisfy $f(z)=0 \Longleftrightarrow f^{\prime}(z) \in\{a, b\}$ for $a / b \in \mathbf{N}$. In this paper we answer this question completely by making use of quasi-normality.

Theorem 1.2. For two distinct nonzero values $a$ and $b$ that satisfy $a / b \in \mathbf{N}$, there is no transcendental meromorphic function $f$ that satisfies $f(z)=0 \Longleftrightarrow f^{\prime}(z) \in$ $\{a, b\}$.

In order to prove Theorem 1.2, we first study the normality or quasi-normality of the family $\mathcal{F}_{a, b}(D)$ which consists of all meromorphic functions $f$ in a plane domain $D \subset \mathbf{C}$ that satisfy $f(z)=0 \Longleftrightarrow f^{\prime}(z) \in\{a, b\}$.

doi:10.5186/aasfm.2013.3832

2010 Mathematics Subject Classification: Primary 30D35, 30D45.

Key words: Meromorphic functions, Bank-Laine functions, quasi-normality, shared values and sets.

*Supported by NNSF of P. R. China (Grant No. 11171045).

†Supported by NNSF of P. R. China (Grant No. 108310904). 
The idea of proving the results in function theory by making use of quasinormality first appears in [7] where it was proved that the derivative of a transcendental meromorphic function with finitely many simple zeros takes every non-zero values infinitely often.

Recall $[3,9]$ that a family $\mathcal{F}$ of meromorphic functions defined in a plane domain $D \subset \mathbf{C}$ is said to be normal (quasi-normal) on $D$, in the sense of Montel, if each sequence $\left\{f_{n}\right\} \subset \mathcal{F}$ contains a subsequence which converges spherically locally uniformly in $D$ (minus a set $E$ that has no accumulation point in $D$ ). The set $E$ may depend on the subsequence. If there exists an integer $\nu \in \mathbf{N}$ such that the set $E$ always can be chosen to contain at most $\nu$ points, then $\mathcal{F}$ is said to be quasi-normal of order $\nu$. Also, we say that the family $\mathcal{F}$ is normal (quasi-normal) at a point $z_{0} \in D$, if there exists a neighborhood $U \subset D$ of $z_{0}$ such that $\mathcal{F}$ is normal (quasi-normal) on $U$. An useful fact, which can be proved by making use of the diagonal method, is that $\mathcal{F}$ is normal (quasi-normal) on $D$ if and only if $\mathcal{F}$ is normal (quasi-normal) at every point in $D$. Another fact is that if $\mathcal{F}$ is not quasi-normal of order $\nu$ in $D$, then there exist a sequence $\left\{f_{n}\right\} \subset \mathcal{F}$ and $\nu+1$ points $z_{1}, z_{2}, \cdots, z_{\nu+1} \in D$ such that no subsequence of $\left\{f_{n}\right\}$ is normal at each $z_{j}$.

The family $\mathcal{F}_{a, b}(D)$ is not quasi-normal in general as showed by the following example.

Example 1. Let for each $n \in \mathbf{N}$

$$
f_{n}(z)=\frac{\sin (n z)}{n}=\frac{e^{i n z}-e^{-i n z}}{2 i n}, \quad(i=\sqrt{-1}) .
$$

Then we have $f_{n}(z)=0 \Longleftrightarrow f_{n}^{\prime}(z) \in\{-1,1\}$. However, it is not difficult to see that no subsequence of $\left\{f_{n}\right\}$ can be normal at every point on the real axis. In fact, for $x_{0} \in \mathbf{R}, f_{n}\left(x_{0}\right) \rightarrow 0$ while $\left|f_{n}\left(x_{0}-\frac{i}{\sqrt{n}}\right)\right| \geq\left(e^{\sqrt{n}}-e^{-\sqrt{n}}\right) /(2 n) \rightarrow \infty$. Hence each subsequence of $\left\{f_{n}\right\}$ fails to be equicontinuous in any neighborhood of $x_{0}$, and so $\mathcal{F}_{-1,1}(\mathbf{C})$ is not quasi-normal.

However, we prove in this paper the following quasi-normality criterion.

Theorem 1.3. For two distinct nonzero values $a$ and $b$ that satisfy $a / b \in \mathbf{N}$, the family $\mathcal{F}_{a, b}(D)$ is quasi-normal on $D$ of order 1.

We remark that if $|\arg a / b| \leq \pi / 3$ and neither $a / b$ nor $b / a$ is an integer, then the family $\mathcal{F}_{a, b}(D)$ is normal in $D$. This can be seen from [2]. We do not know whether the number $\pi / 3$ can be replaced by a larger one.

The following example shows that the conclusion of Theorem 1.3 is sharp.

Example 2. Let $k \in \mathbf{N}$ and for each $n \in \mathbf{N}$

$$
f_{n}(z)=z-\frac{1}{n z^{k}}
$$

Then $\left\{f_{n}\right\}$ is quasi-normal of order 1 on $\mathbf{C}$, since $\left\{f_{n}\right\}$ converges locally uniformly to $z$ on the punctured plane $\mathbf{C} \backslash\{0\}$ and no subsequence is normal at 0 . We see that $f_{n}(z)=0 \Longleftrightarrow f_{n}^{\prime}(z)=k+1$ and $f_{n}^{\prime}(z) \neq 1$. Hence $\left\{f_{n}\right\} \subset \mathcal{F}_{k+1,1}(\mathbf{C})$.

Our proof of Theorem 1.3 is inspired by [7, Theorem 1]. The structure of the present paper is in certain sense similar to [7], and this can be seen by comparing our Lemmas 2.4, 2.7 and 2.8 with Lemmas 4, 7 and 8 in [7], respectively. However, there are many different points and the proofs are different to a large extent. 


\section{Notations and preliminary results}

Throughout in this paper, we denote by $\mathbf{C}$ the complex plane, by $\mathbf{C}^{*}$ the punctured complex plane $\mathbf{C} \backslash\{0\}$, by $\Delta\left(z_{0}, r\right)$ the open disk $\left\{z:\left|z-z_{0}\right|<r\right\}$, by $\Delta^{\circ}\left(z_{0}, r\right)$ the punctured disk $\Delta\left(z_{0}, r\right) \backslash\left\{z_{0}\right\}=\left\{z: 0<\left|z-z_{0}\right|<r\right\}$, and by $\bar{\Delta}\left(z_{0}, r\right)$ the closed disk $\left\{z:\left|z-z_{0}\right| \leq r\right\}$, where $z_{0} \in \mathbf{C}$ and $r>0$.

In Lemmas 2.8, 2.10 and their proofs, and in the proof of Theorem 1.3, we shall use frequently the following auxiliary function

$$
F_{f, a, b}(z):=\frac{f\left(\frac{a+b}{2}+(a-b) z\right)}{a-b}
$$

for a function $f$ and two distinct constants $a$ and $b$.

For a sequence $\left\{f_{n}\right\}$ of functions, we say that they are locally uniformly holomorphic (meromorphic) on $D$ if for each compact subset $E \subset D$, there exists $N \in \mathbf{N}$ such that $f_{n}$ for every $n>N$ is holomorphic (meromorphic) on $E$.

Also, we write $f_{n} \stackrel{\chi}{\rightarrow} f$ on $D$ to indicate that the sequence $\left\{f_{n}\right\}$ converges spherically locally uniformly to $f$ on $D$, and $f_{n} \rightarrow f$ on $D$ if the convergence is already in Euclidean metric. define

Let $f$ be a function meromorphic on $D$. Then for each closed disk $\bar{\Delta}\left(z_{0}, r\right) \subset D$,

$$
A\left(z_{0}, r ; f\right):=\frac{1}{\pi} \iint_{\bar{\Delta}\left(z_{0}, r\right)}\left[f^{\#}(z)\right]^{2} d \sigma .
$$

Here, as usual, $f^{\#}(z)=\left|f^{\prime}(z)\right| /\left(1+|f(z)|^{2}\right)$ is the spherical derivative. An important fact is that $A\left(z_{0}, r ; f\right)$ is the normalized spherical area of the image of $\bar{\Delta}\left(z_{0}, r\right)$ under $f[5]$.

We also use $n(r, f)$ to denote the number of poles of $f$ on $\Delta(0, r)$, counting multiplicity. Similarly, $n(r, 1 / f)$ denotes the number of zeros of $f$ on $\Delta(0, r)$.

For a meromorphic function $f$ on $\mathbf{C}$, its Ahlfors-Shimizu characteristic $[5,10]$ is defined by

$$
T_{0}(r, f)=\int_{0}^{r} \frac{A(0, t ; f)}{t} d t
$$

and the order of $f$ is defined by

$$
\rho(f)=\limsup _{r \rightarrow \infty} \frac{\log ^{+} T_{0}(r, f)}{\log r} .
$$

Thus, each meromorphic function with bounded spherical derivative has order at most 2 .

To prove our results, we require some preliminary results.

Lemma 2.1. [4] Let $\mathcal{F}=\{f\}$ be a family of meromorphic functions on $D$ such that $f(z) \neq 0$ and $f^{\prime}(z) \neq 1$. Then $\mathcal{F}$ is normal on $D$.

Lemma 2.2. [8, Lemma 2] Let $\mathcal{F}$ be a family of meromorphic functions in a domain $D$, and suppose that there exists $A \geq 1$ such that $\left|f^{\prime}(z)\right| \leq A$ whenever $f(z)=0$ and $f \in \mathcal{F}$. Then if $\mathcal{F}$ is not normal at $z_{0}$, there exist,

(a) points $z_{n} \in D, \quad z_{n} \rightarrow z_{0}$;

(b) functions $f_{n} \in \mathcal{F}$; and

(c) positive numbers $\rho_{n} \rightarrow 0$ 
such that $g_{n}(\zeta):=\rho_{n}^{-1} f_{n}\left(z_{n}+\rho_{n} \zeta\right) \stackrel{\chi}{\rightarrow} g(\zeta)$ on $\mathbf{C}$, where $g$ satisfies $g^{\#}(\zeta) \leq g^{\#}(0)=$ $A+1$, and hence is nonconstant and of finite order.

The following lemma is a direct corollary to the maximum modulus principle.

Lemma 2.3. Let $z_{0}$ be a point in $D$ and $\left\{f_{n}\right\}$ be a sequence of meromorphic functions on $D$ such that $f_{n} \stackrel{x}{\rightarrow} f$ on $D \backslash\left\{z_{0}\right\}$, where $f$ may be identically $\infty$.

(a) If $f_{n}$ are holomorphic on $D$ and $f \not \equiv \infty$, then $f_{n} \rightarrow f$ on $D$, and $f$ is holomorphic on $D$;

(b) If $f_{n}$ are zero-free on $D$ and $f \not \equiv 0$, then $f_{n} \stackrel{\chi}{\rightarrow} f$ on $D$, and $f \neq 0$ on $D$;

(c) If $f_{n}$ are holomorphic and zero-free on $D$, then $f_{n} \rightarrow f$ on $D$.

Proof. Since $f_{n} \stackrel{\chi}{\rightarrow} f$ on $D \backslash\left\{z_{0}\right\}, f$ is also meromorphic on $D \backslash\left\{z_{0}\right\}$ or $f \equiv \infty$ [9, Corollary 3.1.4].

Now we turn to prove (a). Since $f_{n}$ is holomorphic, we get $f_{n} \rightarrow f$ on $D \backslash\left\{z_{0}\right\}[9$, Proposition 3.1.6]. Since $f \not \equiv \infty, f$ is also holomorphic on $D \backslash\left\{z_{0}\right\}$ [9, Corollary 3.1.5]. Fix a bounded subdomain $U$ of $D$ such that $z_{0} \in U$ and $\bar{U} \subset D$. Thus for arbitrary given number $\varepsilon>0$, there exists $N \in \mathbf{N}$ such that for $n>N,\left|f_{n}(z)-f(z)\right|<\varepsilon$ on the boundary $\partial U$ of $U$. Hence for $m, n>N,\left|f_{m}(z)-f_{n}(z)\right|<2 \varepsilon$ on $\partial U$. Since $f_{n}$ are holomorphic on $D$, by the maximum modulus principle, we get $\left|f_{m}(z)-f_{n}(z)\right|<2 \varepsilon$ on the domain $\bar{U}$. Thus, by Cauchy's criterion, $\left\{f_{n}\right\}$ converges uniformly to a function $\phi$ which is holomorphic on $U$. Uniqueness of the limit function shows that $f \equiv \phi$ on $U \backslash\left\{z_{0}\right\}$. This shows that $f$ can be extended holomorphicly to $z_{0}$ and hence $f$ is holomorphic on $D$, and $f_{n} \rightarrow f$ on $D$.

Applying (a) to the sequence $\left\{1 / f_{n}\right\}$ and $1 / f$, we prove (b). The (c) is a direct corollary to (a) and (b).

Lemma 2.4. [2, Theorem 4(ii)] Let $k$ be a positive integer. Then the nonconstant meromorphic functions $f \in \mathcal{F}_{k+1,1}(\mathbf{C})$ which are of finite order must be rational functions with the form

$$
f(z)=z-z_{0}-\frac{d}{\left(z-z_{0}\right)^{k}}
$$

where $d$ and $z_{0}$ are constants with $d \neq 0$.

Lemma 2.5. Let $k$ be a positive integer. If the rational function $f$ defined by (1) has two zeros $\pm 1 / 2$, then there exists a $K=K(k)>k+1$ which only depends on $k$ such that

$$
\sup _{z \in \mathbf{C}} f^{\#}(z) \leq K
$$

Proof. Since $f( \pm 1 / 2)=0$, we have

$$
\left(\frac{1}{2}-z_{0}\right)^{k+1}=\left(-\frac{1}{2}-z_{0}\right)^{k+1}=d .
$$

Since $d \neq 0$, we have $z_{0} \neq \pm 1 / 2$. Let $c=\left(\frac{1}{2}-z_{0}\right) /\left(-\frac{1}{2}-z_{0}\right)$. Then $c \neq 1$ and by $(2)$, $c^{k+1}=1$, so that

$$
c \in\left\{c_{j}=\exp \left(\frac{2 j \pi}{k+1} i\right): j=1,2, \cdots, k\right\} .
$$


By $c=\left(\frac{1}{2}-z_{0}\right) /\left(-\frac{1}{2}-z_{0}\right)$, we get $z_{0}=\frac{1+c}{2(1-c)}$, and hence by $(2)$,

$$
d=\left(-\frac{1}{2}-z_{0}\right)^{k+1}=\frac{(-1)^{k+1}}{(1-c)^{k+1}} .
$$

Thus by (1) and (3), $f \in\left\{f_{j}: j=1,2, \cdots, k\right\}$, where

$$
f_{j}(z)=z-\frac{1+c_{j}}{2\left(1-c_{j}\right)}+\frac{(-1)^{k}}{\left(1-c_{j}\right)^{k+1}\left(z-\frac{1+c_{j}}{2\left(1-c_{j}\right)}\right)^{k}} .
$$

Since $f_{j}(z) \rightarrow \infty$ and $f_{j}^{\prime}(z) \rightarrow 1$ as $z \rightarrow \infty$, we have $f_{j}^{\#}(z) \rightarrow 0$ as $z \rightarrow \infty$. Hence, the conclusion follows from the continuity of each $f_{j}^{\#}(z)$ on $\mathbf{C}$.

Lemma 2.6. Let $k$ be a positive integer. Then the sub-family $\mathcal{F}$ of $\mathcal{F}_{k+1,1}(D)$ which consists of holomorphic functions is normal on $D$.

Proof. Suppose that $\mathcal{F}$ is not normal at some point $z_{0} \in D$. Then by Lemma 2.2, there exist functions $\left\{f_{n}\right\} \subset \mathcal{F}$, points $z_{n} \rightarrow z_{0}$ and positive numbers $\rho_{n} \rightarrow 0$ such that $g_{n}(\zeta)=\rho_{n}^{-1} f_{n}\left(z_{n}+\rho_{n} \zeta\right) \rightarrow g(\zeta)$ on $\mathbf{C}$, where $g$ is a nonconstant entire function of finite order and satisfy $g^{\#}(\zeta) \leq g^{\#}(0)=k+2$.

We claim that $g(\zeta)=0 \Longleftrightarrow g^{\prime}(\zeta) \in\{k+1,1\}$.

To prove $g(\zeta)=0 \Longrightarrow g^{\prime}(\zeta) \in\{k+1,1\}$, let $\zeta_{0}$ be a zero of $g$. Then as $g \not \equiv 0$, by Hurwitz's theorem, there exist points $\zeta_{n} \rightarrow \zeta_{0}$ such that $g_{n}\left(\zeta_{n}\right)=0$, and hence $f_{n}\left(z_{n}+\rho_{n} \zeta_{n}\right)=0$. Since $f_{n} \in \mathcal{F}_{k+1,1}(D)$, we get $f_{n}^{\prime}\left(z_{n}+\rho_{n} \zeta_{n}\right) \in\{k+1,1\}$, so that $g_{n}^{\prime}\left(\zeta_{n}\right) \in\{k+1,1\}$. Since $g_{n}^{\prime} \rightarrow g^{\prime}$ on $\mathbf{C}$, we get $g^{\prime}\left(\zeta_{0}\right) \in\{k+1,1\}$. This proves $g(\zeta)=0 \Longrightarrow g^{\prime}(\zeta) \in\{k+1,1\}$.

Now we turn to prove $g^{\prime}(\zeta) \in\{k+1,1\} \Longrightarrow g(\zeta)=0$. let $\zeta_{0}$ be a point such that $g^{\prime}\left(\zeta_{0}\right) \in\{k+1,1\}$. Suppose first that $g^{\prime}\left(\zeta_{0}\right)=k+1$. Since $g^{\#}(0)=k+2$, we have $g^{\prime}(\zeta) \not \equiv k+1$. Thus by $g_{n}^{\prime}(\zeta)-(k+1) \rightarrow g^{\prime}(\zeta)-(k+1)$ and Hurwitz's theorem, there exist points $\zeta_{n} \rightarrow \zeta_{0}$ such that $g_{n}^{\prime}\left(\zeta_{n}\right)=k+1$, and hence $f_{n}^{\prime}\left(z_{n}+\rho_{n} \zeta_{n}\right)=k+1$. Since $f_{n} \in \mathcal{F}_{k+1,1}(D)$, we get $f_{n}\left(z_{n}+\rho_{n} \zeta_{n}\right)=0$, so that $g_{n}\left(\zeta_{n}\right)=0$. Thus by $g_{n} \rightarrow g$, we get $g\left(\zeta_{0}\right)=0$. A similar argument yields that for $g^{\prime}\left(\zeta_{0}\right)=1$, we also have $g\left(\zeta_{0}\right)=0$. This proves $g^{\prime}(\zeta) \in\{k+1,1\} \Longrightarrow g(\zeta)=0$.

Hence the claim is proved. However, by Lemma 2.4, such an entire function $g$ must be a constant. This contradiction shows that $\mathcal{F}$ is normal on $D$.

Lemma 2.7. Let $k$ be a positive integer, and $\left\{f_{n}\right\}$ be a sequence in $\mathcal{F}_{k+1,1}(D)$. Let $z_{0} \in D$. Suppose that

(i) no subsequence of $\left\{f_{n}\right\}$ is normal at $z_{0}$;

(ii) there exists $\delta>0$ such that $f_{n}$ for sufficiently large $n$ has at most one single (simple or multiple) pole in $\Delta\left(z_{0}, \delta\right)$; and

(iii) $\left\{f_{n}\right\}$ is normal on $D \backslash\left\{z_{0}\right\}$.

Then there exists a subsequence of $\left\{f_{n}\right\}$, which we continue to call $\left\{f_{n}\right\}$, such that

(I) $f_{n}(z) \stackrel{\chi}{\rightarrow} z-z_{0}$ on $D \backslash\left\{z_{0}\right\}$;

(II) there exists $\delta_{0}>0$ such that every $f_{n}$ takes each value $w \in \overline{\mathbf{C}}$ at most $k+1$ times on $\Delta\left(z_{0}, \delta_{0}\right)$, counting multiplicity.

Proof. Say $z_{0}=0$. Since $\left\{f_{n}\right\}$ is not normal at $z_{0}=0$, by Lemma 2.2, there exist a subsequence of $\left\{f_{n}\right\}$ which we continue to call $\left\{f_{n}\right\}$, points $z_{n} \rightarrow 0$ and positive 
numbers $\rho_{n} \rightarrow 0$ such that $g_{n}(\zeta)=\rho_{n}^{-1} f_{n}\left(z_{n}+\rho_{n} \zeta\right) \stackrel{\chi}{\rightarrow} g(\zeta)$ on $\mathbf{C}$, where $g$ is a nonconstant meromorphic function of finite order and satisfies $g^{\#}(\zeta) \leq g^{\#}(0)=k+2$. Further, by an argument similar as showed in the proof of Lemma 2.6, we have $g(\zeta)=0 \Longleftrightarrow g^{\prime}(\zeta) \in\{k+1,1\}$. Hence by Lemma 2.4,

$$
g(\zeta)=\zeta-\zeta_{0}-\frac{d}{\left(\zeta-\zeta_{0}\right)^{k}}=\frac{\left(\zeta-\zeta_{0}\right)^{k+1}-d}{\left(\zeta-\zeta_{0}\right)^{k}}
$$

with $d, \zeta_{0} \in \mathbf{C}$ and $d \neq 0$. Let $\zeta_{0}^{(j)}, j=0,1, \cdots, k$, be $k+1$ distinct zeros of g. Then by $(5), \prod_{j=0}^{k}\left(\zeta-\zeta_{0}^{(j)}\right)=P(\zeta):=\left(\zeta-\zeta_{0}\right)^{k+1}-d$, and hence for a given $j_{0} \in\{0,1, \cdots, k\}, \prod_{j \neq j_{0}}\left(\zeta_{0}^{\left(j_{0}\right)}-\zeta_{0}^{(j)}\right)=P^{\prime}\left(\zeta_{0}^{\left(j_{0}\right)}\right)=(k+1)\left(\zeta_{0}^{\left(j_{0}\right)}-\zeta_{0}\right)^{k}$. Thus

$$
\frac{\prod_{j \neq j_{0}}\left(\zeta_{0}^{\left(j_{0}\right)}-\zeta_{0}^{(j)}\right)}{\left(\zeta_{0}^{\left(j_{0}\right)}-\zeta_{0}\right)^{k}}=k+1
$$

for a given $j_{0} \in\{0,1, \cdots, k\}$.

Since $g_{n} \stackrel{x}{\rightarrow} g$ on $\mathbf{C}, g_{n}$ has a pole $\zeta_{n, \infty}$ with $\zeta_{n, \infty} \rightarrow \zeta_{0}$ and $k+1$ zeros $\zeta_{n, 0}^{(j)}$, $(j=0,1, \cdots, k)$, with $\zeta_{n, 0}^{(j)} \rightarrow \zeta_{0}^{(j)}$. Thus $f_{n}$ has a pole $z_{n, \infty}=z_{n}+\rho_{n} \zeta_{n, \infty} \rightarrow 0$ and $k+1$ zeros $z_{n, 0}^{(j)}=z_{n}+\rho_{n} \zeta_{n, 0}^{(j)} \rightarrow 0,(j=0,1, \cdots, k)$.

By the assumption (ii), the multiplicity of the pole $z_{n, \infty}$ is $k$ (for sufficiently large $n)$. Let

$$
f_{n}^{*}(z)=\frac{f_{n}(z)}{R_{n}(z)}, \text { where } R_{n}(z)=\frac{\prod_{j=0}^{k}\left(z-z_{n, 0}^{(j)}\right)}{\left(z-z_{n, \infty}\right)^{k}} .
$$

Then by (ii), $f_{n}^{*}$ for sufficiently large $n$ is holomorphic in $\Delta(0, \delta)$. Let $g_{n}^{*}(\zeta)=$ $f_{n}^{*}\left(z_{n}+\rho_{n} \zeta\right)$. Then $\left\{g_{n}^{*}\right\}$ are locally uniformly holomorphic on C. Since

$$
\rho_{n}^{-1} R_{n}\left(z_{n}+\rho_{n} \zeta\right)=\frac{\prod_{j=0}^{k}\left(\zeta-\zeta_{n, 0}^{(j)}\right)}{\left(\zeta-\zeta_{n, \infty}\right)^{k}} \stackrel{\chi}{\rightarrow} g(\zeta)
$$

on $\mathbf{C}$, and $\rho_{n}^{-1} R_{n}\left(z_{n}+\rho_{n} \zeta\right) g_{n}^{*}(\zeta)=g_{n}(\zeta) \stackrel{\chi}{\rightarrow} g(\zeta)$, we see that $\left\{g_{n}^{*}\right\}$ are locally uniformly zero-free on $\mathbf{C}$, i.e., for each $r>0$, there exists $N \in \mathbf{N}$ such that for $n>N, g_{n}^{*}(\zeta) \neq 0$ on $\bar{\Delta}(0, r)$. We also see that

$$
g_{n}^{*}(\zeta)=f_{n}^{*}\left(z_{n}+\rho_{n} \zeta\right) \rightarrow 1
$$

on $\mathbf{C} \backslash\left\{\zeta_{0}, \zeta_{0}^{(j)}: 0 \leq j \leq k\right\}$, and hence on $\mathbf{C}$ by Lemma 2.3(c). In particular, $f_{n}^{*}\left(z_{n}\right) \neq 0$.

We claim that there exists $\eta, 0<\eta \leq \delta$, such that $f_{n}^{*}$ for sufficiently large $n$ has no zero in $\Delta(0, \eta)$.

Suppose not. Then there exist a subsequence of $\left\{f_{n}^{*}\right\}$, which we continue to call $\left\{f_{n}^{*}\right\}$, and a sequence $\left\{z_{n, 0}\right\}$ of points satisfying $z_{n, 0} \rightarrow 0$ such that $f_{n}^{*}\left(z_{n, 0}\right)=0$. Since $f_{n}^{*}\left(z_{n}\right) \neq 0$, we have $z_{n, 0} \neq z_{n}$. We may say that $z_{n, 0}$ is the nearest zero of $f_{n}^{*}$ away from $z_{n}$, so that $f_{n}^{*}(z) \neq 0$ on $\Delta\left(z_{n},\left|z_{n, 0}-z_{n}\right|\right)$. By (8), we have

$$
\zeta_{n, 0}=\frac{z_{n, 0}-z_{n}}{\rho_{n}} \rightarrow \infty
$$

Set

$$
\hat{f}_{n}^{*}(z)=f_{n}^{*}\left(z_{n}+\left(z_{n, 0}-z_{n}\right) z\right)
$$


Then, $\left\{\hat{f}_{n}^{*}\right\}$ are locally uniformly holomorphic on $\mathbf{C}, \hat{f}_{n}^{*}(z) \neq 0$ on $\Delta(0,1)$, and $\hat{f}_{n}^{*}(1)=0$. Further, let

$$
\hat{f}_{n}(z)=\frac{f_{n}\left(z_{n}+\left(z_{n, 0}-z_{n}\right) z\right)}{z_{n, 0}-z_{n}} .
$$

Then $\hat{f}_{n}(z)=0 \Longleftrightarrow \hat{f}_{n}^{\prime}(z) \in\{k+1,1\}$, since $f_{n}(z)=0 \Longleftrightarrow f_{n}^{\prime}(z) \in\{k+1,1\}$.

By (7), (10) and (11), we see that $\hat{f}_{n}(z)=\hat{R}_{n}(z) \hat{f}_{n}^{*}(z)$, where

$$
\hat{R}_{n}(z)=\frac{R_{n}\left(z_{n}+\left(z_{n, 0}-z_{n}\right) z\right)}{z_{n, 0}-z_{n}}=\frac{\prod_{j=0}^{k}\left(z-\frac{\rho_{n}}{z_{n, 0}-z_{n}} \zeta_{n, 0}^{(j)}\right)}{\left(z-\frac{\rho_{n}}{z_{n, 0}-z_{n}} \zeta_{n, \infty}\right)^{k}} .
$$

Hence by (9) and since $\left\{\hat{f}_{n}^{*}\right\}$ are locally uniformly holomorphic on $\mathbf{C}$, we see that $\left\{\hat{f}_{n}\right\}$ are locally uniformly holomorphic on $\mathbf{C}^{*}$. By (9) and (12), we also see that $\hat{R}_{n}(z) \rightarrow z$ on $\mathbf{C}^{*}$.

Thus, since $\hat{f}_{n}(z)=0 \Longleftrightarrow \hat{f}_{n}^{\prime}(z) \in\{k+1,1\}$ and $\left\{\hat{f}_{n}\right\}$ are locally uniformly holomorphic on $\mathbf{C}^{*}$, by Lemma 2.6, $\left\{\hat{f}_{n}\right\}$ and hence $\left\{\hat{f}_{n}^{*}\right\}$ is normal on $\mathbf{C}^{*}$. Since $\hat{f}_{n}^{*}(1)=0$, by taking a subsequence and renumbering, we may assume that $\hat{f}_{n}^{*} \rightarrow$ $\hat{f}^{*}$ on $\mathbf{C}^{*}$ with $\hat{f}^{*}(1)=0$. As $\left\{\hat{f}_{n}^{*}\right\}$ are locally uniformly holomorphic on $\mathbf{C}$, by Lemma 2.3(a), we get $\hat{f}_{n}^{*} \rightarrow \hat{f}^{*}$ on $\mathbf{C}$ and $\hat{f}^{*}$ is an entire function. Hence, by $\hat{f}_{n}^{*}(0)=f_{n}^{*}\left(z_{n}\right)=g_{n}^{*}(0) \rightarrow 1$, we get $\hat{f}^{*}(0)=1$. Thus $\left(z \hat{f}^{*}(z)\right)^{\prime}-1 \not \equiv 0$. For otherwise, we would have $z \hat{f}^{*}(z)=z+c$ for some constant $c$, which contradicts that $\hat{f}^{*}(1)=0$ and $\hat{f}^{*}(0)=1$.

We claim that $\hat{f}_{n}^{\prime}(z) \neq 1$ on $\Delta(0,1)$ for sufficiently large $n$. Suppose not. Then there exist a subsequence of $\left\{\hat{f}_{n}\right\}$, which we continue to call $\left\{\hat{f}_{n}\right\}$, and a sequence $\left\{z_{n}^{*}\right\}$ of points contained in $\Delta(0,1)$ such that $\hat{f}_{n}^{\prime}\left(z_{n}^{*}\right)=1$. Then, by $\hat{f}_{n}(z)=0 \Longleftrightarrow$ $\hat{f}_{n}^{\prime}(z) \in\{k+1,1\}$, we get $\hat{f}_{n}\left(z_{n}^{*}\right)=0$. Since $\hat{f}_{n}^{*}(z) \neq 0$ on $\Delta(0,1)$, we get from $\hat{f}_{n}(z)=\hat{R}_{n}(z) \hat{f}_{n}^{*}(z)$ that $\hat{R}_{n}\left(z_{n}^{*}\right)=0$. Thus by $(12)$,

$$
z_{n}^{*}=\frac{\rho_{n}}{z_{n, 0}-z_{n}} \zeta_{n, 0}^{\left(j_{0}\right)} \rightarrow 0
$$

for some $j_{0} \in\{0,1, \cdots, k\}$. Hence $\hat{f}_{n}^{*}\left(z_{n}^{*}\right) \rightarrow \hat{f}^{*}(0)=1$ and by $(6)$

$$
\hat{R}_{n}^{\prime}\left(z_{n}^{*}\right)=\frac{\prod_{j \neq j_{0}}\left(\zeta_{n, 0}^{\left(j_{0}\right)}-\zeta_{n, 0}^{(j)}\right)}{\left(\zeta_{n, 0}^{\left(j_{0}\right)}-\zeta_{n, \infty}\right)^{k}} \rightarrow \frac{\prod_{j \neq j_{0}}\left(\zeta_{0}^{\left(j_{0}\right)}-\zeta_{0}^{(j)}\right)}{\left(\zeta_{0}^{\left(j_{0}\right)}-\zeta_{0}\right)^{k}}=k+1 .
$$

Thus by $\hat{f}_{n}(z)=\hat{R}_{n}(z) \hat{f}_{n}^{*}(z)$, we get a contradiction:

$$
1=\hat{f}_{n}^{\prime}\left(z_{n}^{*}\right)=\hat{R}_{n}^{\prime}\left(z_{n}^{*}\right) \hat{f}_{n}^{*}\left(z_{n}^{*}\right)+\hat{R}_{n}\left(z_{n}^{*}\right) \hat{f}_{n}^{*^{\prime}}\left(z_{n}^{*}\right)=\hat{R}_{n}^{\prime}\left(z_{n}^{*}\right) \hat{f}_{n}^{*}\left(z_{n}^{*}\right) \rightarrow k+1 .
$$

This contradiction shows that $\hat{f}_{n}^{\prime}(z) \neq 1$ on $\Delta(0,1)$.

Since $\hat{f}_{n}(z)=\hat{R}_{n}(z) \hat{f}_{n}^{*}(z) \rightarrow z \hat{f}^{*}(z)$ on $\mathbf{C}^{*}$, we get $\hat{f}_{n}^{\prime}(z)-1 \rightarrow\left(z \hat{f}^{*}(z)\right)^{\prime}-1$ on $\mathrm{C}^{*}$. Since $\left(z \hat{f}^{*}(z)\right)^{\prime}-1 \not \equiv 0$ and $\hat{f}_{n}^{\prime}(z)-1 \neq 0$ on $\Delta(0,1)$, by Lemma 2.3(b), we have $\hat{f}_{n}^{\prime}(z)-1 \stackrel{\chi}{\rightarrow}\left(z \hat{f}^{*}(z)\right)^{\prime}-1$ on $\Delta(0,1)$, and $\left(z \hat{f}^{*}(z)\right)^{\prime}-1 \neq 0$ on $\Delta(0,1)$. However, we have $\left.\left(z \hat{f}^{*}(z)\right)^{\prime}\right|_{z=0}=\hat{f}^{*}(0)=1$. This is a contradiction.

Thus $f_{n}^{*}$ for sufficiently large $n$ is zero-free and holomorphic in $\Delta(0, \eta)$. Since $\left\{f_{n}^{*}\right\}$ is normal on $D \backslash\{0\}$, it follows from Lemma 2.3(c) that $\left\{f_{n}^{*}\right\}$ is normal at 0 and 
hence is normal on $D$. Further, as $f_{n}^{*}\left(z_{n}\right)=g_{n}^{*}(0) \rightarrow 1$, there exists a subsequence of $\left\{f_{n}^{*}\right\}$, which we continue to call $\left\{f_{n}^{*}\right\}$, such that $f_{n}^{*} \stackrel{\chi}{\rightarrow} f^{*}$ on $D$ with $f^{*}(0)=1$.

Now we claim that there exists $\mu>0$ such that $f_{n}^{\prime}(z) \neq 1$ in $\Delta(0, \mu)$ for sufficiently large $n$. Suppose not. Then there exist a subsequence of $\left\{f_{n}\right\}$, which we continue to call $\left\{f_{n}\right\}$, and a sequence $\left\{w_{n}\right\}$ of points satisfying $w_{n} \rightarrow 0$ such that $f_{n}^{\prime}\left(w_{n}\right)=1$. Then by the condition $f_{n}(z)=0 \Longleftrightarrow f_{n}^{\prime}(z) \in\{k+1,1\}$, we see that $f_{n}\left(w_{n}\right)=0$ and hence $R_{n}\left(w_{n}\right)=0$ since $f_{n}=R_{n} f_{n}^{*}$ and $f_{n}^{*}$ is zero-free in $\Delta(0, \eta)$. Thus by (7), $w_{n}=z_{n, 0}^{\left(j_{0}\right)}$ for some $j_{0} \in\{0,1, \cdots, k\}$. Hence we have $f_{n}^{*}\left(w_{n}\right) \rightarrow f^{*}(0)=1$, and by $(6)$

$$
\begin{aligned}
R_{n}^{\prime}\left(w_{n}\right)= & R_{n}^{\prime}\left(z_{n, 0}^{\left(j_{0}\right)}\right)=\frac{\prod_{j \neq j_{0}}\left(z_{n, 0}^{\left(j_{0}\right)}-z_{n, 0}^{(j)}\right)}{\left(z_{n, 0}^{\left(j_{0}\right)}-z_{n, \infty}\right)^{k}} \\
= & \frac{\prod_{j \neq j_{0}}\left(\zeta_{n, 0}^{\left(j_{0}\right)}-\zeta_{n, 0}^{(j)}\right)}{\left(\zeta_{n, 0}^{\left(j_{0}\right)}-\zeta_{n, \infty}\right)^{k}} \rightarrow \frac{\prod_{j \neq j_{0}}\left(\zeta_{0}^{\left(j_{0}\right)}-\zeta_{0}^{(j)}\right)}{\left(\zeta_{0}^{\left(j_{0}\right)}-\zeta_{0}\right)^{k}}=k+1 .
\end{aligned}
$$

Thus we get by $f_{n}=R_{n} f_{n}^{*}$ the following contradiction:

$$
1=f_{n}^{\prime}\left(w_{n}\right)=R_{n}^{\prime}\left(w_{n}\right) f_{n}^{*}\left(w_{n}\right)+R_{n}\left(w_{n}\right) f_{n}^{* \prime}\left(w_{n}\right)=R_{n}^{\prime}\left(w_{n}\right) f_{n}^{*}\left(w_{n}\right) \rightarrow k+1 .
$$

This proved the claim that for some $\mu>0, f_{n}^{\prime}(z) \neq 1$ in $\Delta(0, \mu)$ for sufficiently large $n$.

Next we prove that $\left(z f^{*}(z)\right)^{\prime} \equiv 1$. Suppose not. Since $f_{n}^{*}$ for sufficiently large $n$ is zero-free and holomorphic in $\Delta(0, \eta)$, we have by Lemma 2.3 (a) that $f_{n}^{*} \rightarrow f^{*}$ on $\Delta(0, \eta)$ with $f^{*}(0)=1$. It follows that $f_{n}(z)=R_{n}(z) f_{n}^{*}(z) \rightarrow z f^{*}(z)$ on $\Delta^{\circ}(0, \eta)$. Thus $f_{n}^{\prime}(z) \rightarrow\left(z f^{*}(z)\right)^{\prime}$ and hence $f_{n}^{\prime}(z)-1 \rightarrow\left(z f^{*}(z)\right)^{\prime}-1$ on $\Delta^{\circ}(0, \eta)$. Since $f_{n}^{\prime} \neq 1$ in $\Delta(0, \mu)$, we may say that $f_{n}^{\prime}-1 \neq 0$ on $\Delta(0, \eta)$. Thus by Lemma 2.3(b), we get $f_{n}^{\prime}(z)-1 \stackrel{\chi}{\rightarrow}\left(z f^{*}(z)\right)^{\prime}-1$ on $\Delta(0, \eta)$, and $\left(z f^{*}(z)\right)^{\prime}-1 \neq 0$ on $\Delta(0, \eta)$. This contradicts that $\left.\left(z f^{*}(z)\right)^{\prime}\right|_{z=0}=f^{*}(0)=1$.

Thus $\left(z f^{*}(z)\right)^{\prime} \equiv 1$ and hence $z f^{*}(z)=z+c$ for some constant $c$. Since $f^{*}(0)=1$, we get $c=0$, and hence $f^{*}(z) \equiv 1$. Thus, by $R_{n}(z) \rightarrow z$ in $\mathbf{C}^{*}$ and $f_{n}^{*} \stackrel{\chi}{\rightarrow} 1$ in $D$, we get $f_{n}(z)=R_{n}(z) f_{n}^{*}(z) \stackrel{\chi}{\rightarrow} z$ in $D \backslash\{0\}$. The assertion (I) for the special case $z_{0}=0$ is proved. In general case, one can consider the sequence $\left\{F_{n}\right\}$, where $F_{n}(z)=f_{n}\left(z_{0}+z\right)$ and $z \in U=\left\{z-z_{0}: z \in D\right\}$, and obtain that $F_{n}(z) \stackrel{\chi}{\rightarrow} z$ in $U \backslash\{0\}$ by the special case. Then by the fact $f_{n}(z)=F_{n}\left(z-z_{0}\right)$, the assertion (I) follows.

Now we turn to prove the assertion (II). Fix a number $\delta_{0}>0$ such that $\bar{\Delta}\left(0, \delta_{0}\right) \subset$ $D$. Then as $f_{n}=R_{n} f_{n}^{*}$ and $f_{n}^{*} \rightarrow f^{*} \equiv 1$ on $\bar{\Delta}\left(0, \delta_{0}\right)$, there exists $N_{1} \in \mathbf{N}$ such that for $n>N_{1}, f_{n}$ has exactly one pole with multiplicity $k$ on $\bar{\Delta}\left(0, \delta_{0}\right) \subset D$ and $f_{n} \neq 0, \infty$ on the circle $|z|=\delta_{0}$. In other words, every $f_{n}$ for $n>N_{1}$ takes the value $\infty$ exactly $k$ times on $\bar{\Delta}\left(0, \delta_{0}\right)$.

For $w \neq \infty$, we consider two cases. Suppose first that $|w| \leq \delta_{0} / 2$. By (I), we have $f_{n}(z) \stackrel{\chi}{\rightarrow} z$ on $D \backslash\{0\}$, and hence on the circle $|z|=\delta_{0}, f_{n}(z) \rightarrow z, f_{n}^{\prime}(z)-1 \rightarrow 0$ and $z f_{n}^{\prime}(z)-f_{n}(z) \rightarrow 0$. Thus there exists $N_{2} \in \mathbf{N}, N_{2} \geq N_{1}$, such that for $n>N_{2}$ and the points $z$ on $|z|=\delta_{0},\left|f_{n}(z)\right| \geq 3 \delta_{0} / 4,\left|f_{n}^{\prime}(z)-1\right| \leq \delta_{0} /\left(16+8 \delta_{0}\right)$ and $\left|z f_{n}^{\prime}(z)-f_{n}(z)\right| \leq \delta_{0} /\left(16+8 \delta_{0}\right)$. It follows from the argument principle that for 
$n>N_{2}$,

$$
\begin{aligned}
& \left|n\left(\delta_{0}, \frac{1}{f_{n}-w}\right)-n\left(\delta_{0}, f_{n}-w\right)-1\right| \\
& =\left|\frac{1}{2 \pi i} \int_{|z|=\delta_{0}} \frac{f_{n}^{\prime}(z)}{f_{n}(z)-w} d z-\frac{1}{2 \pi i} \int_{|z|=\delta_{0}} \frac{1}{z-w} d z\right| \\
& =\frac{1}{2 \pi}\left|\int_{|z|=\delta_{0}} \frac{z f_{n}^{\prime}(z)-f_{n}(z)-w\left(f_{n}^{\prime}(z)-1\right)}{\left(f_{n}(z)-w\right)(z-w)} d z\right| \\
& \leq \frac{1}{2 \pi} \int_{|z|=\delta_{0}} \frac{\frac{\delta_{0}}{16+8 \delta_{0}}\left(1+\frac{\delta_{0}}{2}\right)}{\left(\frac{3}{4} \delta_{0}-\frac{1}{2} \delta_{0}\right)\left(\delta_{0}-\frac{1}{2} \delta_{0}\right)}|d z|=\frac{1}{2},
\end{aligned}
$$

and hence $n\left(\delta_{0}, \frac{1}{f_{n}-w}\right)-n\left(\delta_{0}, f_{n}-w\right)-1=0$ since it is an integer. Thus $n\left(\delta_{0}, \frac{1}{f_{n}-w}\right)$ $=n\left(\delta_{0}, f_{n}-w\right)+1=n\left(\delta_{0}, f_{n}\right)+1=k+1$. That is to say, every $f_{n}$ for $n>N_{2}$ takes each value $w$ satisfying $|w| \leq \delta_{0} / 2$ exactly $k+1$ times on $\bar{\Delta}\left(0, \delta_{0}\right) \subset D$.

Suppose now that $|w| \geq \delta_{0} / 2$. Similar as showed above, we have $f_{n}(z) \rightarrow z$, $f_{n}^{\prime}(z)-1 \rightarrow 0$ and $z f_{n}^{\prime}(z)-f_{n}(z) \rightarrow 0$ on the circle $|z|=\delta_{0} / 4$. Thus there exists $N_{3} \in \mathbf{N}, N_{3} \geq N_{1}$, such that for $n>N_{3}$ and the points $z$ on $|z|=\delta_{0} / 4,\left|f_{n}(z)\right| \leq$ $3 \delta_{0} / 8,\left|f_{n}^{\prime}(z)-1\right| \leq \delta_{0} /\left(16+8 \delta_{0}\right)$ and $\left|z f_{n}^{\prime}(z)-f_{n}(z)\right| \leq \delta_{0} /\left(16+8 \delta_{0}\right)$. It follows from the argument principle that for $n>N_{3}$,

$$
\begin{aligned}
& \left|n\left(\frac{\delta_{0}}{4}, \frac{1}{f_{n}-w}\right)-n\left(\frac{\delta_{0}}{4}, f_{n}-w\right)\right| \\
& =\left|\frac{1}{2 \pi i} \int_{|z|=\frac{\delta_{0}}{4}} \frac{f_{n}^{\prime}(z)}{f_{n}(z)-w} d z-\frac{1}{2 \pi i} \int_{|z|=\frac{\delta_{0}}{4}} \frac{1}{z-w} d z\right| \\
& =\frac{1}{2 \pi}\left|\int_{|z|=\frac{\delta_{0}}{4}} \frac{z f_{n}^{\prime}(z)-f_{n}(z)-w\left(f_{n}^{\prime}(z)-1\right)}{\left(f_{n}(z)-w\right)(z-w)} d z\right| \\
& =\frac{1}{2 \pi}\left|\int_{|z|=\frac{\delta_{0}}{4}} \frac{\frac{1}{w}\left(z f_{n}^{\prime}(z)-f_{n}(z)\right)-\left(f_{n}^{\prime}(z)-1\right)}{\left(w-f_{n}(z)\right)\left(1-\frac{z}{w}\right)} d z\right| \\
& \leq \frac{1}{2 \pi} \int_{|z|=\frac{\delta_{0}}{4}} \frac{\frac{\delta_{0}}{16+8 \delta_{0}}\left(\frac{2}{\delta_{0}}+1\right)}{\left(\frac{1}{2} \delta_{0}-\frac{3}{8} \delta_{0}\right)\left(1-\frac{1}{2}\right)}|d z|=\frac{1}{2},
\end{aligned}
$$

and hence $n\left(\frac{\delta_{0}}{4}, \frac{1}{f_{n}-w}\right)=n\left(\frac{\delta_{0}}{4}, f_{n}-w\right)=n\left(\frac{\delta_{0}}{4}, f_{n}\right) \leq n\left(\delta_{0}, f_{n}\right)=k$. That is to say, every $f_{n}$ for $n>N_{3}$ takes each value $w$ satisfying $|w| \geq \delta_{0} / 2$ at most $k$ times on $\bar{\Delta}\left(0, \delta_{0} / 4\right) \subset D$.

Thus for $n>N=\max \left\{N_{1}, N_{2}, N_{3}\right\}$, every $f_{n}$ takes each value $w \in \overline{\mathbf{C}}$ on $\bar{\Delta}\left(0, \delta_{0} / 4\right)$ at most $k+1$ times.

The proof of Lemma 2.7 is completed.

Lemma 2.8. Let $k$ be a positive integer, and $\left\{f_{n}\right\}$ be a sequence in $\mathcal{F}_{k+1,1}(D)$. Let $z_{0} \in D$. Suppose that

(i) no subsequence of $\left\{f_{n}\right\}$ is normal at $z_{0}$; and

(ii) for every $\delta>0$, there exists $N \in \mathbf{N}$ such that $f_{n}$ for $n>N$ has at least two distinct poles in $\Delta\left(z_{0}, \delta\right)$. 
Then there exist a subsequence of $\left\{f_{n}\right\}$ which we continue to call $\left\{f_{n}\right\}$, and a sequence $\left\{\eta_{n}\right\}$ of positive numbers satisfying $\eta_{n} \rightarrow 0$ such that $f_{n}$ has at least two distinct zeros $a_{n}$ and $b_{n}$ in $\Delta\left(z_{0}, \eta_{n}\right)$ such that

$$
\sup _{\bar{\Delta}(0,1)} F_{f_{n}, a_{n}, b_{n}}^{\#}(z) \rightarrow \infty .
$$

Proof. Say $z_{0}=0$. As showed in the proof of Lemma 2.7, since $\left\{f_{n}\right\}$ is not normal at 0 , there exist a subsequence of $\left\{f_{n}\right\}$ which we continue to call $\left\{f_{n}\right\}$, points $z_{n} \rightarrow 0$ and positive numbers $\rho_{n} \rightarrow 0$ such that

$$
g_{n}(\zeta)=\rho_{n}^{-1} f_{n}\left(z_{n}+\rho_{n} \zeta\right) \stackrel{x}{\rightarrow} g(\zeta)=\zeta-\zeta_{0}-\frac{d}{\left(\zeta-\zeta_{0}\right)^{k}}=\frac{\left(\zeta-\zeta_{0}\right)^{k+1}-d}{\left(\zeta-\zeta_{0}\right)^{k}}
$$

on $\mathbf{C}$, where $d, \zeta_{0} \in \mathbf{C}$ and $d \neq 0$.

As showed in the proof of Lemma 2.7, it follows that $f_{n}$ has a pole $z_{n, \infty}=z_{n}+$ $\rho_{n} \zeta_{n, \infty} \rightarrow 0$ with $\zeta_{n, \infty} \rightarrow \zeta_{0}$ and $k+1$ zeros $z_{n, 0}^{(j)}=z_{n}+\rho_{n} \zeta_{n, 0}^{(j)} \rightarrow 0,(j=0,1, \cdots, k)$, with $\zeta_{n, 0}^{(j)} \rightarrow \zeta_{0}^{(j)}$, where $\zeta_{0}^{(j)}$ are $k+1$ distinct zeros of $g$. Note that, we also have (6).

We claim that $g_{n}^{\prime}(\zeta) \neq 1$ locally uniformly on $\mathbf{C}$, i.e., for each $r>0$, there exists $N \in \mathbf{N}$ such that for $n>N, g_{n}^{\prime}(\zeta) \neq 1$ on $\bar{\Delta}(0, r)$. Suppose not, then there exist $r_{0}>0$, a subsequence of $\left\{g_{n}\right\}$, which we continue to call $\left\{g_{n}\right\}$, and a sequence $\left\{\zeta_{n}\right\}$ of points contained in $\bar{\Delta}\left(0, r_{0}\right)$ such that $g_{n}^{\prime}\left(\zeta_{n}\right)=1$. By taking a subsequence, we may say $\zeta_{n} \rightarrow c \in \bar{\Delta}\left(0, r_{0}\right)$. By $g_{n}^{\prime}\left(\zeta_{n}\right)=1$, we get $f_{n}^{\prime}\left(z_{n}+\rho_{n} \zeta_{n}\right)=1$, and hence by the condition, $f_{n}\left(z_{n}+\rho_{n} \zeta_{n}\right)=0$. Thus $g_{n}\left(\zeta_{n}\right)=0$ and hence $g(c)=0$ by $g_{n} \stackrel{\chi}{\rightarrow} g$, so that $g$ and hence $g_{n}$ (for sufficiently large $n$ ) are holomorphic in a neighborhood of $c$. It follows that $g_{n} \rightarrow g$ and hence $g_{n}^{\prime} \rightarrow g^{\prime}$ on this neighborhood. Thus by $g_{n}^{\prime}\left(\zeta_{n}\right)=1$, we get $g^{\prime}(c)=1$. This is a contradiction, since $g^{\prime}(\zeta) \neq 1$ on $\mathbf{C}$.

Since $g_{n} \stackrel{\chi}{\rightarrow} g$ on $\mathbf{C}$ and $\zeta_{0}$ is a pole of $g$ with exact multiplicity $k$, every $g_{n}$ (for sufficiently large $n$ ) has exactly $k$ poles tending to $\zeta_{0}$.

We claim that the $k$ poles of $g_{n}$ coincide. Suppose not. Then $g_{n}$ has $s \geq 2$ distinct poles $\zeta_{n, \infty}^{(j)}, 1 \leq j \leq s$ with multiplicity $m_{j}$ such that $\sum_{j=1}^{s} m_{j}=k$ and $\zeta_{n, \infty}^{(j)} \rightarrow \zeta_{0}$. By taking subsequence, we may assume that the number $s$ and the multiplicities $m_{j}$ are all independent of $n$. Further, as $\zeta_{0}$ is the unique pole of $g$, the poles of $g_{n}$ other than $\zeta_{n, \infty}^{(j)}, 1 \leq j \leq s$, if exist, must tend to $\infty$. Also, the zeros of $g_{n}$ other than $\zeta_{n, 0}^{(j)}, 0 \leq j \leq k$, if exist, must tend to $\infty$. It follows that the sequence $\left\{g_{n}^{*}\right\}$ of functions defined by

$$
g_{n}^{*}(\zeta):=\frac{\prod_{j=1}^{s}\left(\zeta-\zeta_{n, \infty}^{(j)}\right)^{m_{j}}}{\prod_{j=0}^{k}\left(\zeta-\zeta_{n, 0}^{(j)}\right)} g_{n}(\zeta)
$$

is locally uniformly zero-free and holomorphic on C. Since $g_{n} \stackrel{\chi}{\rightarrow} g$ on $\mathbf{C}$, we see from (16) that

$$
g_{n}^{*}(\zeta) \rightarrow 1
$$

on $\mathbf{C} \backslash\left\{\zeta_{0}, \zeta_{0}^{(j)}: 0 \leq j \leq k\right\}$, and hence on $\mathbf{C}$ by Lemma 2.3(c).

By (16), we have

$$
g_{n}(\zeta)=\frac{\prod_{j=0}^{k}\left(\zeta-\zeta_{n, 0}^{(j)}\right)}{\prod_{j=1}^{s}\left(\zeta-\zeta_{n, \infty}^{(j)}\right)^{m_{j}}} g_{n}^{*}(\zeta)
$$


and hence

$$
g_{n}^{\prime}(\zeta)=\frac{\left(g_{n}^{*}(\zeta) \prod_{j=0}^{k}\left(\zeta-\zeta_{n, 0}^{(j)}\right)\right)^{\prime}}{\prod_{j=1}^{s}\left(\zeta-\zeta_{n, \infty}^{(j)}\right)^{m_{j}}}-g_{n}^{*}(\zeta) \prod_{j=0}^{k}\left(\zeta-\zeta_{n, 0}^{(j)}\right) \frac{\sum_{j=1}^{s} m_{j} \prod_{i \neq j}\left(\zeta-\zeta_{n, \infty}^{(i)}\right)}{\prod_{j=1}^{s}\left(\zeta-\zeta_{n, \infty}^{(j)}\right)^{m_{j}+1}}
$$

so that

$$
g_{n}^{\prime}(\zeta)-1=\frac{M_{n}(\zeta)}{\prod_{j=1}^{s}\left(\zeta-\zeta_{n, \infty}^{(j)}\right)^{m_{j}+1}},
$$

where

$$
\begin{aligned}
M_{n}(\zeta)= & \left(g_{n}^{*}(\zeta) \prod_{j=0}^{k}\left(\zeta-\zeta_{n, 0}^{(j)}\right)\right)^{\prime} \prod_{j=1}^{s}\left(\zeta-\zeta_{n, \infty}^{(j)}\right) \\
& -g_{n}^{*}(\zeta) \prod_{j=0}^{k}\left(\zeta-\zeta_{n, 0}^{(j)}\right) \sum_{j=1}^{s} m_{j} \prod_{i \neq j}\left(\zeta-\zeta_{n, \infty}^{(i)}\right)-\prod_{j=1}^{s}\left(\zeta-\zeta_{n, \infty}^{(j)}\right)^{m_{j}+1} .
\end{aligned}
$$

Since $\zeta_{n, 0}^{(j)} \rightarrow \zeta_{0}^{(j)},(0 \leq j \leq k)$ and $\zeta_{n, \infty}^{(j)} \rightarrow \zeta_{0},(1 \leq j \leq s)$, we see from (17) and (19) that

$$
\begin{aligned}
M_{n}(\zeta) \rightarrow & (k+1)\left(\zeta-\zeta_{0}\right)^{k+s}-\left[\left(\zeta-\zeta_{0}\right)^{k+1}-d\right] \cdot k\left(\zeta-\zeta_{0}\right)^{s-1}-\left(\zeta-\zeta_{0}\right)^{k+s} \\
& =k d\left(\zeta-\zeta_{0}\right)^{s-1}
\end{aligned}
$$

on C. Thus, by Hurwitz's theorem, $M_{n}$ has $s-1 \geq 1$ zeros (counting multiplicity) tending to $\zeta_{0}$.

On the other hand, we can see from (19) that for each $1 \leq \nu \leq s$,

$$
M_{n}\left(\zeta_{n, \infty}^{(\nu)}\right)=-g_{n}^{*}\left(\zeta_{n, \infty}^{(\nu)}\right) \prod_{j=0}^{k}\left(\zeta_{n, \infty}^{(\nu)}-\zeta_{n, 0}^{(j)}\right) \cdot m_{\nu} \prod_{i \neq \nu}\left(\zeta_{n, \infty}^{(\nu)}-\zeta_{n, \infty}^{(i)}\right) \neq 0 .
$$

Hence, the $s-1 \geq 1$ zeros of $M_{n}$ are different from the points $\left\{\zeta_{n, \infty}^{(j)}: 1 \leq j \leq s\right\}$, so that by $(18), g_{n}^{\prime}(\zeta)-1$ has $s-1 \geq 1$ zeros tending to $\zeta_{0}$. This contradicts the above claim that $g_{n}^{\prime}(\zeta) \neq 1$ locally uniformly on $\mathbf{C}$.

This contradiction shows that the $k$ poles of $g_{n}$ coincide, and hence $\zeta_{n, \infty}$ is a pole of $g_{n}$ with exact multiplicity $k$. Thus by (15), $z_{n, \infty}=z_{n}+\rho_{n} \zeta_{n, \infty}$ is a pole of $f_{n}$ with exact multiplicity $k$.

Now let

$$
\begin{aligned}
R_{n}(z) & =\frac{\prod_{j=0}^{k}\left(z-z_{n, 0}^{(j)}\right)}{\left(z-z_{n, \infty}\right)^{k}}, \\
f_{n}^{*}(z) & =\frac{f_{n}(z)}{R_{n}(z)}, \\
g_{n}^{*}(\zeta) & =f_{n}^{*}\left(z_{n}+\rho_{n} \zeta\right)=\frac{\left(\zeta-\zeta_{n, \infty}\right)^{k}}{\prod_{j=0}^{k}\left(\zeta-\zeta_{n, 0}^{(j)}\right)} g_{n}(\zeta) .
\end{aligned}
$$

Then as showed above, by (15) and (22), we have

$$
g_{n}^{*}(\zeta) \rightarrow 1
$$

on $\mathbf{C} \backslash\left\{\zeta_{0}, \zeta_{0}^{(j)}: 0 \leq j \leq k\right\}$, and hence on $\mathbf{C}$ by Lemma 2.3(c). 
We claim that for every $\delta>0$, there exists $N \in \mathbf{N}$ such that $f_{n}^{*}$ for $n>N$ has at least one zero in $\Delta(0, \delta)$.

Suppose not, then there exist $\delta_{0}>0$ and a subsequence of $\left\{f_{n}^{*}\right\}$, which we continue to call $\left\{f_{n}^{*}\right\}$, such that $f_{n}^{*} \neq 0$ on $\Delta\left(0, \delta_{0}\right)$. Since $f_{n}=R_{n} f_{n}^{*}$ and $f_{n}(z)=0$ $\Longleftrightarrow f_{n}^{\prime}(z) \in\{1, k+1\}$, we see by $(20)$ that $f_{n}(z) \neq 0$ and $f_{n}^{\prime}(z) \neq 1$ for $z \in$ $\Delta\left(0, \delta_{0}\right) \backslash\left\{z_{n, 0}^{(j)}: 0 \leq j \leq k\right\}$. Since $z_{n, 0}^{(j)} \rightarrow 0$, by Gu's criterion (Lemma 2.1), $\left\{f_{n}\right\}$ and hence $\left\{f_{n}^{*}\right\}$ is normal on $\Delta^{\circ}\left(0, \delta_{0}\right)$. So we may assume that $f_{n}^{*} \stackrel{\chi}{\rightarrow} f^{*}$ on $\Delta^{\circ}\left(0, \delta_{0}\right)$, where $f^{*}$ may be $\infty$ identically.

We prove further that there exists $0<\delta_{1} \leq \delta_{0}$ such that $f_{n}^{\prime}(z) \neq 1$ on $\Delta\left(0, \delta_{1}\right)$ for sufficiently large $n$. Suppose not, then as showed in the proof of Lemma 2.7 (before (13)), there exists a subsequence of $\left\{f_{n}\right\}$, which we continue to call $\left\{f_{n}\right\}$, such that $f_{n}^{\prime}\left(z_{n, 0}^{\left(j_{0}\right)}\right)=1$ for some $j_{0}$. This, combined with (21), (23) and (6), would lead to the following contradiction:

$$
\begin{aligned}
1 & =f_{n}^{\prime}\left(z_{n, 0}^{\left(j_{0}\right)}\right)=R_{n}^{\prime}\left(z_{n, 0}^{\left(j_{0}\right)}\right) f_{n}^{*}\left(z_{n, 0}^{\left(j_{0}\right)}\right)=\frac{\prod_{j \neq j_{0}}\left(z_{n, 0}^{\left(j_{0}\right)}-z_{n, 0}^{(j)}\right)}{\left(z_{n, 0}^{\left(j_{0}\right)}-z_{n, \infty}\right)^{k}} f_{n}^{*}\left(z_{n, 0}^{\left(j_{0}\right)}\right) \\
= & \frac{\prod_{j \neq j_{0}}\left(\zeta_{n, 0}^{\left(j_{0}\right)}-\zeta_{n, 0}^{(j)}\right)}{\left(\zeta_{n, 0}^{\left(j_{0}\right)}-\zeta_{n, \infty}\right)^{k}} g_{n}^{*}\left(\zeta_{n, 0}^{\left(j_{0}\right)}\right) \rightarrow \frac{\prod_{j \neq j_{0}}\left(\zeta_{0}^{\left(j_{0}\right)}-\zeta_{0}^{(j)}\right)}{\left(\zeta_{0}^{\left(j_{0}\right)}-\zeta_{0}\right)^{k}}=k+1 .
\end{aligned}
$$

We claim that $f^{*} \not \equiv 0$. For otherwise, we would have $f_{n} \stackrel{\chi}{\rightarrow} 0$ and hence $f_{n}^{\prime} \rightarrow 0$, $f_{n}^{\prime \prime} \rightarrow 0$ on $\Delta^{\circ}\left(0, \delta_{1}\right)$. Thus by the argument principle and $f_{n}^{\prime}(z) \neq 1$ on $\Delta\left(0, \delta_{1}\right)$,

$$
n\left(\frac{\delta_{1}}{2}, f_{n}^{\prime}-1\right)=\left|n\left(\frac{\delta_{1}}{2}, f_{n}^{\prime}-1\right)-n\left(\frac{\delta_{1}}{2}, \frac{1}{f_{n}^{\prime}-1}\right)\right|=\frac{1}{2 \pi}\left|\int_{|z|=\frac{\delta_{1}}{2}} \frac{f_{n}^{\prime \prime}}{f_{n}^{\prime}-1} d z\right| \rightarrow 0 .
$$

It follows that $f_{n}^{\prime}$ has no poles in $\Delta\left(0, \delta_{1} / 2\right)$. This is a contradiction, since $z_{n, \infty} \rightarrow 0$ is a pole of $f_{n}$.

Thus $f^{*} \not \equiv 0$. Hence by $f_{n}^{*} \neq 0$ on $\Delta\left(0, \delta_{0}\right)$ and $f_{n}^{*} \stackrel{\chi}{\rightarrow} f^{*}$ on $\Delta^{\circ}\left(0, \delta_{0}\right)$, it follows from Lemma 2.3(b) that $f_{n}^{*} \stackrel{\chi}{\rightarrow} f^{*}$ on $\Delta\left(0, \delta_{0}\right)$. Since $f_{n}^{*}\left(z_{n}\right)=g_{n}^{*}(0) \rightarrow 1$, we get $f^{*}(0)=1$. This shows that $f^{*}$ is holomorphic in some neighborhood $\Delta\left(0, \delta_{2}\right)$ of 0 and hence so is $f_{n}^{*}$ in $\Delta\left(0, \delta_{2} / 2\right)$ for sufficiently large $n$. Thus by $f_{n}=R_{n} f_{n}^{*}, f_{n}$ has only one single pole (of multiplicity $k$ ) in $\Delta\left(0, \delta_{2} / 2\right)$. This contradicts the assumption (ii).

Thus, for every $\delta>0$, there exists $N \in \mathbf{N}$ such that $f_{n}^{*}$ for $n>N$ has at least one zero in $\Delta(0, \delta)$. It follows that there exists a subsequence of $\left\{f_{n}^{*}\right\}$, which we continue to call $\left\{f_{n}^{*}\right\}$, such that $f_{n}^{*}$ has a zero $b_{n}$ in $\Delta(0,1 / n)$. By (22) and (23), we have

$$
\zeta_{n}^{*}=\frac{b_{n}-z_{n}}{\rho_{n}} \rightarrow \infty
$$

Set $a_{n}=z_{n, 0}^{(1)}$ and $\eta_{n}=\left|z_{n, 0}^{(1)}\right|+1 / n$. Then by $z_{n, \infty}=z_{n}+\rho_{n} \zeta_{n, \infty}$ with $\zeta_{n, \infty} \rightarrow \zeta_{0}$ and $a_{n}=z_{n}+\rho_{n} \zeta_{n, 0}^{(1)}$ with $\zeta_{n, 0}^{(1)} \rightarrow \zeta_{0}^{(1)}$, we see that $\eta_{n} \rightarrow 0, a_{n}, b_{n} \in \Delta\left(0, \eta_{n}\right)$ and from $(24)$ that $a_{n} \neq b_{n}$ and

$$
\frac{z_{n, \infty}-\frac{a_{n}+b_{n}}{2}}{a_{n}-b_{n}}=\frac{2 \zeta_{n, \infty}-\zeta_{n, 0}^{(1)}-\zeta_{n}^{*}}{2\left(\zeta_{n, 0}^{(1)}-\zeta_{n}^{*}\right)} \rightarrow \frac{1}{2}
$$


This, combined with $F_{f_{n}, a_{n}, b_{n}}(1 / 2)=f_{n}\left(a_{n}\right) /\left(a_{n}-b_{n}\right)=0$ and

$$
F_{f_{n}, a_{n}, b_{n}}\left(\frac{z_{n, \infty}-\frac{a_{n}+b_{n}}{2}}{a_{n}-b_{n}}\right)=\frac{f_{n}\left(z_{n, \infty}\right)}{a_{n}-b_{n}}=\infty,
$$

shows that each subsequence of $\left\{F_{f_{n}, a_{n}, b_{n}}\right\}$ fails to be equicontinuous in any neighborhood of $z=1 / 2$ and hence fails to be normal at $1 / 2$. Now (14) follows from Marty's theorem. Lemma 2.8 is proved.

Lemma 2.9. If $f$ is a meromorphic function of infinite order, then there exist points $z_{n} \rightarrow \infty$ and positive numbers $\varepsilon_{n} \rightarrow 0$ such that

$$
A\left(z_{n}, \varepsilon_{n} ; f\right)=\frac{1}{\pi} \iint_{\bar{\Delta}\left(z_{n}, \varepsilon_{n}\right)}\left(f^{\#}(z)\right)^{2} d \sigma \rightarrow \infty .
$$

Proof. See [7, p. 12].

Lemma 2.10. Let $k$ be a positive integer, and $f$ be a meromorphic function in $\mathcal{F}_{k+1,1}(\mathbf{C})$. If $f$ is of infinite order, then $f$ has infinitely many pairs of distinct zeros $\left(z_{n, 1}, z_{n, 2}\right)$ such that $z_{n, 1}-z_{n, 2} \rightarrow 0$ and

$$
\sup _{\bar{\Delta}(0,1)} F_{f, z_{n, 1}, z_{n, 2}}(z) \rightarrow \infty \text {. }
$$

Proof. Since $f$ is of infinite order, by Lemma 2.9, we have (25) for some points $z_{n} \rightarrow \infty$ and positive numbers $\varepsilon_{n} \rightarrow 0$. It follows that there exist $w_{n} \in \bar{\Delta}\left(z_{n}, \varepsilon_{n}\right)$ such that $f^{\#}\left(w_{n}\right) \rightarrow \infty$. Thus, by Marty's theorem, no subsequence of $\left\{f_{n}\right\}$ is normal at 0 , where

$$
f_{n}(z)=f\left(w_{n}+z\right) .
$$

Thus, by Lemma 2.6, $f_{n}$ for sufficiently large $n$ has at least one pole $w_{n}$ such that $w_{n} \rightarrow w_{0}$.

Suppose first that there exist $\delta>0$ and $N \in \mathbf{N}$ such that $f_{n}$ for $n>N$ has at most one single (simple or multiple) pole in $\Delta(0, \delta)$. Then $f_{n}$ for $n>N$ is holomorphic on $\Delta(0, \delta) \backslash\left\{w_{n}\right\}$. Since $w_{n} \rightarrow w_{0}$, it follows from Lemma 2.6 that $\left\{f_{n}\right\}$ is normal on $\Delta^{\circ}(0, \delta)$. So by Lemma 2.7 , there exists a subsequence of $\left\{f_{n}\right\}$, which we continue to call $\left\{f_{n}\right\}$, such that for some $\delta_{0}>0, f_{n}$ takes each value $w \in \overline{\mathbf{C}}$ at most $k+1$ times on $\Delta\left(0, \delta_{0}\right)$, counting multiplicity. Thus for sufficiently large $n$,

$$
A\left(z_{n}, \varepsilon_{n} ; f\right) \leq A\left(0, \delta_{0} ; f_{n}\right) \leq k+1
$$

which contradicts $(25)$.

Thus there exists a subsequence of $\left\{f_{n}\right\}$, which we continue to call $\left\{f_{n}\right\}$, such that for every $\delta>0$, there exists $N \in \mathbf{N}$ such that $f_{n}$ for $n>N$ has at least two distinct poles in $\Delta(0, \delta)$. Then by Lemma 2.8, there exists a subsequence of $\left\{f_{n}\right\}$, which we continue to call $\left\{f_{n}\right\}$, such that each $f_{n}$ has at least two distinct zeros $a_{n}$ and $b_{n}$ tending to 0 such that

$$
\sup _{\bar{\Delta}(0,1)} F_{f_{n}, a_{n}, b_{n}}^{\#}(z) \rightarrow \infty .
$$

Let $z_{n, 1}=w_{n}+a_{n}, z_{n, 2}=w_{n}+b_{n}$. Then $\left(z_{n, 1}, z_{n, 2}\right)$ is a pair of distinct zeros of $f$ satisfying $z_{n, 1}-z_{n, 2} \rightarrow 0$, and (26) follows from (28), since $F_{f, z_{n, 1}, z_{n, 2}}(z) \equiv F_{f_{n}, a_{n}, b_{n}}(z)$ by $(27)$. The lemma is proved. 


\section{Proof of Theorem 1.3}

By the definition, we have $\mathcal{F}_{a, b}=b \mathcal{F}_{a / b, 1}=\left\{b f: f \in \mathcal{F}_{a / b, 1}\right\}$ for $b \neq 0$. Thus by the assumption that $a / b$ is a positive integer and $a \neq b$, we may assume that $a=k+1$ and $b=1$, where $k$ is a positive integer.

Let $E \subset D$ be the set of points at which $\mathcal{F}_{k+1,1}(D)$ is not normal. Then for every $z_{0} \in E$, there exists at least one sequence $\left\{f_{n}\right\} \subset \mathcal{F}_{k+1,1}(D)$ such that no subsequence of $\left\{f_{n}\right\}$ is normal at $z_{0}$. We consider two cases.

Case 1. For every $z_{0} \in E$ and every sequence $\left\{f_{n}\right\} \subset \mathcal{F}_{k+1,1}(D)$ which has no subsequence normal at $z_{0}$, there exist $\delta>0$ and $N \in \mathbf{N}$ such that $f_{n}$ for $n>N$ has at most one single (simple or multiple) pole in $\Delta\left(z_{0}, \delta\right)$.

We first show that $\mathcal{F}_{k+1,1}(D)$ is quasi-normal at every point $w_{0} \in D$.

To prove this, let $\left\{f_{n}\right\} \subset \mathcal{F}_{k+1,1}(D)$ be a sequence. If $w_{0} \notin E$, the by the definition of $E,\left\{f_{n}\right\}$ is normal at $w_{0}$; If $w_{0} \in E$, there are two cases: one is that $\left\{f_{n}\right\}$ has subsequence which is normal at $w_{0}$, and the other is that no subsequence is normal at $w_{0}$. In the later case, by Lemma $2.6, f_{n}$ for sufficiently large $n$ has at least one pole $w_{n}$ such that $w_{n} \rightarrow w_{0}$. Hence, by the hypothesis, there exists $\delta>0$ such that $f_{n}$ for sufficiently large $n$ is holomorphic in $\Delta\left(w_{0}, \delta\right) \backslash\left\{w_{n}\right\}$. Thus, again by Lemma 2.6, $\left\{f_{n}\right\}$ is normal on $\Delta^{\circ}\left(w_{0}, \delta\right)$. These discussions show that every sequence $\left\{f_{n}\right\}$ has a subsequence which is normal on $\Delta^{\circ}\left(w_{0}, \eta\right)$ for some $\eta>0$. Thus, by the definition, $\mathcal{F}_{k+1,1}(D)$ is quasi-normal at every point in $D$. Hence, $\mathcal{F}_{k+1,1}(D)$ is quasi-normal on $D$.

Next, we show further that $\mathcal{F}_{k+1,1}(D)$ is quasi-normal of order 1 .

To prove this, let $\left\{f_{n}\right\} \subset \mathcal{F}_{k+1,1}(D)$ be a sequence. Since $\mathcal{F}_{k+1,1}(D)$ is quasinormal on $D$, there exists a subsequence of $\left\{f_{n}\right\}$, which we continue to call $\left\{f_{n}\right\}$, and a set $E_{0}$ having no accumulation points in $D$ such that $f_{n} \stackrel{\chi}{\rightarrow} \phi$ on $D \backslash E_{0}$.

We have to show that the set $E_{0}$ can be chosen to be a single-point set. Suppose not. Then there exist two distinct points $z_{1}$ and $z_{2}$ such that no subsequence of $\left\{f_{n}\right\}$ is normal at $z_{1}$ or $z_{2}$. Then by Lemma 2.7 , the limit function $\phi$ coincides with $z-z_{1}$ in a punctured neighborhood of $z_{1}$ and with $z-z_{2}$ in a punctured neighborhood of $z_{2}$. It follows from the uniqueness of the limit function that $z-z_{1} \equiv z-z_{2}$. This is impossible, since $z_{1} \neq z_{2}$.

Case 2. There exist $z_{0} \in E$ and a sequence $\left\{f_{n}\right\} \subset \mathcal{F}_{k+1,1}(D)$ which has no subsequence normal at $z_{0}$ such that for every $\delta>0$, there exists $N \in \mathbf{N}$ such that $f_{n}$ for $n>N$ has at least two distinct poles in $\Delta\left(z_{0}, \delta\right)$.

We argue by contradictions for showing that this case can not occur.

By Lemma 2.8, there exists a subsequence of $\left\{f_{n}\right\}$, which we continue to call $\left\{f_{n}\right\}$, such that each $f_{n}$ (for sufficiently large $n$ ) has at least two distinct zeros $u_{n}$ and $v_{n}$ tending to $z_{0}$ as $n \rightarrow \infty$ such that

$$
\sup _{\bar{\Delta}(0,1)} F_{f_{n}, u_{n}, v_{n}}^{\#}(z)>K+1
$$

where the constant $K>k+1$ is defined in Lemma 2.5.

Fix $\delta>0$ such that $\bar{\Delta}\left(z_{0}, 3 \delta\right) \subset D$. It guarantees that for each pair $(a, b)$ of distinct zeros of $f_{n}$ in $\Delta\left(z_{0}, \delta\right)$, the corresponding function $F_{f_{n}, a, b}(z)$ is meromorphic on $\bar{\Delta}(0,1)$. Since $f_{n} \not \equiv 0$, each $f_{n}$ has finitely many zeros in $\Delta\left(z_{0}, \delta\right)$. This, combined 
with (29), shows that the set

$$
E_{n}=\left\{(a, b): a, b \in f_{n}^{-1}(0) \cap \Delta\left(z_{0}, \delta\right) \text { satisfying } \sup _{\frac{\Delta}{\Delta}(0,1)} F_{f_{n}, a, b}^{\#}(z)>K+1\right\}
$$

is non-empty and finite. For $(a, b) \in E_{n}$, define

$$
\tau(a, b):=\frac{|a-b|}{\delta-\left|\frac{a+b}{2}-z_{0}\right|}>0 .
$$

Then there exists $\left(a_{n}, b_{n}\right) \in E_{n}$ such that for every $(a, b) \in E_{n}$,

$$
\tau_{n}:=\tau\left(a_{n}, b_{n}\right) \leq \tau(a, b) .
$$

Since $\left(u_{n}, v_{n}\right) \in E_{n}$, we have $\tau_{n} \leq \tau\left(u_{n}, v_{n}\right) \rightarrow 0$ and hence $a_{n}-b_{n} \rightarrow 0$.

Let $h_{n}(z)=F_{f_{n}, a_{n}, b_{n}}(z)$. Then by $a_{n}-b_{n} \rightarrow 0$, the sequence $\left\{h_{n}\right\}$ are locally uniformly meromorphic on $\mathbf{C}$. And by the definition of the set $E_{n}$, we have

$$
\sup _{\bar{\Delta}(0,1)} h_{n}^{\#}(z)>K+1 \text {. }
$$

We claim that no subsequence of $\left\{h_{n}\right\}$ is normal on C. Suppose not, by taking a subsequence, we may say $h_{n} \stackrel{\chi}{\rightarrow} h$ on C. Since $h_{n}( \pm 1 / 2)=0$, we have $h( \pm 1 / 2)=0$ so that $h \not \equiv \infty$. Thus by (33),

$$
\sup _{\bar{\Delta}(0,1)} h^{\#}(z) \geq K+1 .
$$

It then follows that $h$ is nonconstant and from $K>k+1$ that $h^{\prime}(z) \not \equiv 1$ and $h^{\prime}(z) \not \equiv k+1$.

Since $f_{n}(z)=0 \Longleftrightarrow f_{n}^{\prime}(z) \in\{1, k+1\}$, we have $h_{n}(z)=0 \Longleftrightarrow h_{n}^{\prime}(z) \in\{1, k+1\}$. Hence, by Hurwitz's theorem, $h(z)=0 \Longleftrightarrow h^{\prime}(z) \in\{1, k+1\}$.

We claim that $h$ is of infinite order. Suppose not, then as $h$ is nonconstant, it follows from Lemma 2.4 that $h$ is a rational function with the form (1). Hence, as $h( \pm 1 / 2)=0$, we get by Lemma 2.5 that $\sup _{\bar{\Delta}(0,1)} h^{\#}(z) \leq K$. This contradicts (34).

Thus, $h$ is of infinite order. Hence, by Lemma 2.10 , there exist two distinct zeros $\alpha$ and $\beta$ of $h$ which are not 0 such that $|\alpha-\beta|<1$ and

$$
\sup _{\bar{\Delta}(0,1)} F_{h, \alpha, \beta}^{\#}(z)>K+2 \text {. }
$$

Since $h_{n} \stackrel{\chi}{\rightarrow} h$ on $\mathbf{C}$, there exist points $\alpha_{n} \rightarrow \alpha$ and $\beta_{n} \rightarrow \beta$ such that $h_{n}\left(\alpha_{n}\right)=$ $h_{n}\left(\beta_{n}\right)=0$, and by (35), for sufficiently large $n$,

$$
\sup _{\bar{\Delta}(0,1)} F_{h_{n}, \alpha_{n}, \beta_{n}}^{\#}(z)>K+1 .
$$

Now set

$$
\hat{a}_{n}=\frac{a_{n}+b_{n}}{2}+\left(a_{n}-b_{n}\right) \alpha_{n}, \quad \hat{b}_{n}=\frac{a_{n}+b_{n}}{2}+\left(a_{n}-b_{n}\right) \beta_{n} .
$$

Then $\hat{a}_{n}$ and $\hat{b}_{n}$ are two distinct zeros of $f_{n}$ by the definition of $h_{n}$. Since $\tau_{n} \rightarrow 0$ and $\alpha_{n} \rightarrow \alpha$, we have

$$
\left|\hat{a}_{n}-z_{0}\right| \leq\left|\frac{a_{n}+b_{n}}{2}-z_{0}\right|+\left|a_{n}-b_{n}\right|\left|\alpha_{n}\right|=\delta-\left(\frac{1}{\tau_{n}}-\left|\alpha_{n}\right|\right)\left|a_{n}-b_{n}\right|<\delta
$$

for sufficiently large $n$. Thus $\hat{a}_{n} \in \Delta\left(z_{0}, \delta\right)$. Similarly, $\hat{b}_{n} \in \Delta\left(z_{0}, \delta\right)$. 
Further, as $F_{f_{n}, \hat{a}_{n}, \hat{b}_{n}}(z) \equiv F_{h_{n}, \alpha_{n}, \beta_{n}}(z)$, we get by (36) that

$$
\sup _{\bar{\Delta}(0,1)} F_{f_{n}, \hat{a}_{n}, \hat{b}_{n}}^{\#}(z)>K+1 \text {. }
$$

Thus $\left(\hat{a}_{n}, \hat{b}_{n}\right) \in E_{n}$ by (30), and hence $\tau\left(a_{n}, b_{n}\right) \leq \tau\left(\hat{a}_{n}, \hat{b}_{n}\right)$ by $(32)$.

However, by (31) and (37), we have

$$
\begin{aligned}
\frac{\tau\left(\hat{a}_{n}, \hat{b}_{n}\right)}{\tau\left(a_{n}, b_{n}\right)} & =\frac{\delta-\left|\frac{a_{n}+b_{n}}{2}-z_{0}\right|}{\delta-\left|\frac{a_{n}+b_{n}}{2}-z_{0}+\frac{\alpha_{n}+\beta_{n}}{2}\left(a_{n}-b_{n}\right)\right|}\left|\alpha_{n}-\beta_{n}\right| \\
& \leq \frac{\delta-\left|\frac{a_{n}+b_{n}}{2}-z_{0}\right|}{\delta-\left|\frac{a_{n}+b_{n}}{2}-z_{0}\right|-\left|\frac{\alpha_{n}+\beta_{n}}{2}\right|\left|a_{n}-b_{n}\right|}\left|\alpha_{n}-\beta_{n}\right| \\
& =\frac{\left|\alpha_{n}-\beta_{n}\right|}{1-\left|\frac{\alpha_{n}+\beta_{n}}{2}\right| \tau_{n}} \rightarrow|\alpha-\beta|<1,
\end{aligned}
$$

so that for sufficiently large $n, \tau\left(\hat{a}_{n}, \hat{b}_{n}\right)<\tau\left(a_{n}, b_{n}\right)$. This contradicts that $\tau\left(a_{n}, b_{n}\right) \leq$ $\tau\left(\hat{a}_{n}, \hat{b}_{n}\right)$.

Thus no subsequence of $\left\{h_{n}\right\}$ is normal on $\mathbf{C}$. Now let $H$ be the set of points at which $\left\{h_{n}\right\}$ is not normal. Then $H$ is non-empty.

Suppose first that for every $\zeta_{0} \in H$ and every subsequence $\left\{h_{n_{j}}\right\}$ of $\left\{h_{n}\right\}$ which has no subsequence normal at $\zeta_{0}$, there exist $\eta>0$ and $J \in \mathbf{N}$ such that $h_{n_{j}}$ for $j>J$ has at most one single (simple or multiple) pole in $\Delta\left(\zeta_{0}, \eta\right)$.

Then by an argument similar to that in Case $1,\left\{h_{n}\right\}$ is quasinormal of order 1 on C, and further, there exists a subsequence of $\left\{h_{n}\right\}$, which we continue to call $\left\{h_{n}\right\}$, and a point $\zeta_{0}^{*} \in H$ such that $h_{n}(z) \stackrel{\chi}{\rightarrow} z-\zeta_{0}^{*}$ on $\mathbf{C} \backslash\left\{\zeta_{0}^{*}\right\}$. But, this contradicts $h_{n}( \pm 1 / 2)=0$.

Thus there exist $\zeta_{0}^{* *} \in H$ and a subsequence $\left\{h_{n_{j}}\right\}$ of $\left\{h_{n}\right\}$ which has no subsequence normal at $\zeta_{0}^{* *}$ such that for every $\eta>0$, there exists $J \in \mathbf{N}$ such that $h_{n_{j}}$ for $j>J$ has at least two distinct poles in $\Delta\left(\zeta_{0}^{* *}, \eta\right)$. We rewrite the subsequence $\left\{h_{n_{j}}\right\}$ by $\left\{h_{n}\right\}$.

Then by Lemma 2.8, there exists a subsequence of $\left\{h_{n}\right\}$, which we continue to call $\left\{h_{n}\right\}$, such that each $h_{n}$ has at least two distinct zeros $a_{n}^{*}$ and $b_{n}^{*}$ tending to $\zeta_{0}^{* *}$ as $n \rightarrow \infty$ such that

$$
\sup _{\bar{\Delta}(0,1)} F_{h_{n}, a_{n}^{*}, b_{n}^{*}}^{\#}(z)>K+1 .
$$

Now let

$$
A_{n}=\frac{a_{n}+b_{n}}{2}+\left(a_{n}-b_{n}\right) a_{n}^{*}, \quad B_{n}=\frac{a_{n}+b_{n}}{2}+\left(a_{n}-b_{n}\right) b_{n}^{*} .
$$

Then as showed above, $A_{n}$ and $B_{n}$ are two distinct zeros of $f_{n}$ in $\Delta\left(z_{0}, \delta\right)$, and by (39) with the fact $F_{f_{n}, A_{n}, B_{n}}(z) \equiv F_{h_{n}, a_{n}^{*}, b_{n}^{*}}(z)$,

$$
\sup _{\bar{\Delta}(0,1)} F_{f_{n}, A_{n}, B_{n}}^{\#}(z)>K+1 .
$$

It follows that $\left(A_{n}, B_{n}\right) \in E_{n}$ and hence $\tau\left(a_{n}, b_{n}\right) \leq \tau\left(A_{n}, B_{n}\right)$ by (32). However, an argument similar to (38) yields that $\tau\left(A_{n}, B_{n}\right)<\tau\left(a_{n}, b_{n}\right)$ for sufficiently large $n$, which contradicts that $\tau\left(a_{n}, b_{n}\right) \leq \tau\left(A_{n}, B_{n}\right)$.

The proof of Theorem 1.3 is completed. 


\section{Proof of Theorem 1.2}

As $a / b$ is a positive integer and $a, b$ are distinct and nonzero, we may assume that $\{a, b\}=\{k+1,1\}$, where $k$ is a positive integer.

Suppose that there is a transcendental meromorphic function $f$ in $\mathcal{F}_{k+1,1}(\mathbf{C})$. By Theorem A, the function $f$ is of infinite order.

Thus by Lemma 2.9, there exist points $z_{n} \rightarrow \infty$ and positive numbers $\varepsilon_{n} \rightarrow 0$ such that

$$
A\left(z_{n}, \varepsilon_{n} ; f\right)=\frac{1}{\pi} \iint_{\bar{\Delta}\left(z_{n}, \varepsilon_{n}\right)}\left(f^{\#}(z)\right)^{2} d \sigma \rightarrow \infty .
$$

It follows that there exist $w_{n} \in \bar{\Delta}\left(z_{n}, \varepsilon_{n}\right), w_{n} \rightarrow \infty$, such that $f^{\#}\left(w_{n}\right) \rightarrow \infty$, and hence by Marty's theorem, no subsequence of $\left\{f_{n}\right\}$ is normal at 0 , where $f_{n}(z)=$ $f\left(w_{n}+z\right)$.

Since $f \in \mathcal{F}_{k+1,1}(\mathbf{C})$, we see that $\left\{f_{n}\right\} \subset \mathcal{F}_{k+1,1}(\mathbf{C})$. Thus by Theorem 1.3, $\left\{f_{n}\right\}$ is quasi-normal of order 1 on $\mathbf{C}$. Since no subsequence of $\left\{f_{n}\right\}$ is normal at 0 , $\left\{f_{n}\right\}$ is normal on $\mathbf{C}^{*}$. Further, by the proof of Theorem 1.3, only the Case 1 can occur. It follows that there exist $\delta>0$ and $N \in \mathbf{N}$ such that $f_{n}$ for $n>N$ has at most one single (simple or multiple) pole in $\Delta(0, \delta)$. So by Lemma $2.7(\mathrm{II})$, there exists a subsequence of $\left\{f_{n}\right\}$, which we continue to call $\left\{f_{n}\right\}$, such that on some neighborhood $\bar{\Delta}\left(0, \delta_{0}\right)$ of 0 , each $f_{n}$ takes each value $a \in \overline{\mathbf{C}}$ at most $k+1$ times, counting multiplicity. Thus $A\left(z_{n}, \varepsilon_{n} ; f\right) \leq A\left(0, \delta_{0} ; f_{n}\right) \leq k+1$. This contradicts (40).

The proof of Theorem 1.2 is completed.

Acknowledgement. The authors are grateful to the referee for his/her valuable suggestions and comments, which help a lot in improving the paper.

\section{References}

[1] Bank, S., and I. Laine: On the oscillation theory of $f^{\prime \prime}+A f=0$ where A is entire. - Trans. Amer. Math. Soc. 273, 1982, 351-363.

[2] Chang, J. M., and Y.F. WAng: Shared values, Picard values and vormality. - Tohoku Math. J. 63, 2011, 149-162.

[3] Chunang, C. T.: Normal families of meromorphic functions. - World Scientific, 1993.

[4] GU, Y.X.: A criterion for normality of families of meromorphic functions. - Sci. Sinica, special issue 1 on Math., 1979, 267-274 (in Chinese).

[5] Hayman, W. K.: Meromorphic functions. - Clarendon Press, Oxford, 1964.

[6] Laine, I.: Nevanlinna theory and complex differential equations. - de Gruyter Stud. Math. 15, Walter de Gruyter, Berlin, 1993.

[7] Nevo, S., X. C. PAng, and L. Zalcman: Quasinormality and meromorphic functions with multiple zeros. - J. Anal. Math. 101, 2007, 1-23.

[8] Pang, X. C., and L. Zalcman: Normal families and shared values. - Bull. London Math. Soc. 32, 2000, 325-331.

[9] Schiff, J.: Normal families. - Springer-Verlag, Berlin, 1993.

[10] YANG, L.: Value distribution theory. - Springer-Verlag, Berlin, 1993.

Received 9 September 2011 • First revised received 25 January 2012 • Second revised received 11 September 2012 • Accepted 12 December 2012 